\title{
Time for a Comprehensive Ultrasound- Enhanced Trauma Management
}

In the last few years, a large number of papers have reported new and interesting applications of ultrasound (US) in trauma management. US in trauma is, nowadays, well beyond focused assessment by sonography for trauma (FAST), the first historical standardized US approach to polytrauma, that could be really considered as the 'mother' of emergency US. FAST represents the paradigm of point-of-care critical US and is probably the simplest way to start training: a key answer to a specific question is required at that moment in a particular setting, bedside US findings give in a short time a 'yes/no' confirmation about the clinical suspicion, and the doctor can immediately decide how he/she can proceed (treatment, further imaging, etc.).

If clinical reasoning (and not US pictures) is your instrument, even a doubtful US finding is helpful to you and your patient.

This simple approach carries a revolutionary concept. US is no longer only an organ-based examination, but in critical and emergency settings, it has become a problem-based diagnostic tool. For this reason, US probes are today in the hands of doctors taking care of acute patients, everywhere they need a critical clinical answer, and not only in radiological suites.

There is a kind of reluctance for many surgeons to accept this change.

The papers included in this 'Focus on' could help us to understand the value, the limits, and the requirements for performing US in trauma, from the prehospital setting to the follow-up. Ruesseler et al. outline the impact of on-scene US in the prehospital management and triage of trauma patients [1]. Relying on their previous studies, they demonstrate how a systematic use of US by the rescue team could affect the destination of the patient and the subsequent hospital organization.

Beyond FAST and understanding the problembased approach, it should be easy to appreciate the

Eur J Trauma Emerg Surg 2009;35:339-40

DOI 10.1007/s00068-009-3004-3

Published Online: August 4, 2009 paper of Breitkreutz et al. on basic cardiac US for surgeons [2]. In a simple but very rigorous way, this paper shows and demonstrates that the heart is not an affair for cardiosurgeons only, but for acute care surgeons too. Facing a critical surgical patient, both in the emergency department (ED) and in the ward or intensive care unit (ICU), it is crucial to know if your patient is empty or full looking at the cardiac chambers and at the inferior vena cava (IVC) caliper; if right chambers are dilated in your patient in cardiac arrest on the fifth postoperative day after femoral nailing for trauma (or Hartmann procedure for left anorectal trauma): if so, pulmonary embolism is probably the correct diagnosis and you need a thrombolytic agent more than adrenaline during cardiopulmonary resuscitation (CPR).

The detection of fractures by US is not a new application, but what is new is the inclusion of this application in a systematic approach, integrated in the primary and secondary survey. This is explained in detail by Al-Kadi et al. [3], with clear and intuitive pictures.

The fact that focused point-of-care US has gained interest in trauma management does not mean that organ-based 'classical' echography should be abandoned. A typical use is the follow-up of solid organ injuries managed non-operatively. In this situation, more experienced ultrasonographers are required to follow the evolution of the lesions. Padalino et al. [4] report on a long US follow-up study for the nonoperative management (NOM) of blunt liver trauma. US seems to be useful in allowing an earlier resumption of activities, tailored to the individual patient and not to fixed protocols.

There are many other explored and emerging applications of US in trauma not included in this 'Focus on.'

Airway management can be made safer in some situations by US, from the quick assessment of the correct positioning of an endotracheal tube to the placement of a tracheostomy in a short or injured neck [5].

In thoracic trauma, extended-FAST (EFAST) allows to rule in or rule out the hemothorax and pneumothorax in a few seconds, during primary survey [6]. 
The significance of that is extraordinary, as the surgeon can recognize the clinical problem even before the standard chest X-ray, treat it if needed, skipping over the chest X-ray in some situations [7].

The detection of sternal and rib fractures is easy, with a sensitivity higher than standard X-rays [8]. Pulmonary contusion becomes apparent on US early after trauma at the moment of EFAST examination and can be monitored in the ICU over the following days [9].

The recent experiences with second-generation contrast media in solid organ injuries [10] revealed very promising, and in some centers, a modified early follow-up for NOM is under evaluation.

The interpretation of optic nerve sheet caliper as a marker of increased intracranial pressure is also an investigational field [11], like the role of US monitoring of volume replacement and hemodynamic assessment in the ED and ICU [12].

We cannot forget the role of interventional US in both major and minor trauma (venous and arterial cannulation, nerve blocks for fracture reduction, diagnostic peritoneal aspiration for suspected hollow viscus lesion, etc.).

It is absolutely clear that we are witnessing the inclusion of US in every phase of the ABCDE assessment of the trauma patient.

An emerging concept is US-enhanced trauma resuscitation and management.

To achieve this goal, there is a strong requirement for education, training, and competence-based credentials. There is a growing literature and wide debate on defining the standards for training and the certification of competence [13]. The scientific societies involved in the care of critical patients are called upon to give their contribution and the European Society for Trauma and Emergency Surgery (ESTES) is ready for that.

In the meantime, there is no reason to postpone the programs of training. It is useful to remember a citation from Lucas Greiner, past President of the European Federation of Societies for Ultrasound in Medicine and Biology (EFSUMB): "There is more risk in not doing US than in doing it, provided the brain is connected to the hand-held probe..."

One of the specificities and a fascinating part of acute care surgery is to make decisions in a short time for critically ill patients. The more precise are the information available, the more effective and decisive the clinical choice. US can give answers to the surgeon without any delay, sometimes avoiding time-consuming procedures, provided he/she can immediately rely on the result of US. In other words, the acute care surgeons of the future should have US in their own hands! Don't fear, you can do that. Don't let others take the lead.

$$
\begin{array}{r}
\text { Mauro Zago, MD } \\
\text { Emergency and Trauma Surgery Section } \\
\text { General and Minimally Invasive Surgery Department } \\
\text { Istituto Clinico Humanitas } \\
\text { Rozzano, (Milano) - Italy }
\end{array}
$$

\section{References}

1. Ruesseler M, Kirschning T, Breitkreutz, R, Marzi I, Walcher F. Prehospital and emergency department ultrasound in blunt trauma. Eur J Trauma Emerg Surg 2009;35:341-6.

2. Breitkreutz R, Walcher F, Ilper $H$, Seeger FH, Via G, Steiger $H$. Focused echocardiography in life support: the subcostal window. What the surgeon should know for critical care applications. Eur J Trauma Emerg Surg 2009;35:347-56.

3. Al-Kadi AS, Gillman LM, Ball CG, Panebianco N, Kirkpatrick AW. Resuscitative long-bone sonography for the clinician: usefulness and pitfalls of focused clinical ultrasound to detect longbone fractures during trauma resuscitation. Eur J Trauma Emerg Surg 2009;35:357-63.

4. Padalino P, Bomben F, Chiara O, Montagnolo G, Marini A, Zago $M$, Rebora P. Healing of blunt liver injury after non-operative management: role of ultrasonography follow-up. Eur J Trauma Emerg Surg 2009;35:364-70.

5. Sustić A. Role of ultrasound in the airway management of critically ill patients. Crit Care Med 2007;35:S173-7.

6. Kirkpatrick AW, Sirois M, Laupland KB, Liu D, Rowan K, Ball CG, Hameed SM, Brown R, Simons R, Dulchavsky SA, Hamiilton DR, Nicolaou $S$. Hand-held thoracic sonography for detecting posttraumatic pneumothoraces: the extended focused assessment with sonography for trauma (EFAST). J Trauma 2004;57:288-95.

7. Zago M, Casamassima A, Kurihara H, Mariani D, Turconi MG, Bertocco D, Pelosi L, Rosati R. E-FAST and CX-Ray in trauma patients: something can change. Int J Disaster Med 2006;4:6-7

8. Jin W, Yang DM, Kim HC, Ryu KN. Diagnostic values of sonography for assessment of sternal fractures compared with conventional radiography and bone scans. J Ultrasound Med 2006;25:1263-8.

9. Soldati G, Testa A, Silva FR, Carbone L, Portale G, Silveri NG. Chest ultrasonography in lung contusion. Chest 2006;130:533-8.

10. Catalano O, Aiani L, Barozzi L, Bokor D, De Marchi A, Faletti C, Maggioni F, Montanari N, Orlandi PE, Siani A, Sidhu PS, Thompson PK, Valentino M, Ziosi A, Martegani A. CEUS in abdominal trauma: multi-center study. Abdom Imaging 2009;34:225-34.

11. Le A, Hoehn ME, Smith ME, Spentzas T, Schlappy D, Pershad J. Bedside sonographic measurement of optic nerve sheath diameter as a predictor of increased intracranial pressure in children. Ann Emerg Med 2009;53:785-91.

12. Carr BG, Dean AJ, Everett WW, Ku BS, Mark DG, Okusanya O, Horan AD, Gracias VH. Intensivist bedside ultrasound (INBU) for volume assessment in the intensive care unit: a pilot study. J Trauma 2007;63:495-500.

13. Neri L, Storti E, Lichtenstein D. Toward an ultrasound curriculum for critical care medicine. Crit Care Med 2007;35:S290-304 